\title{
Dental development of children and adolescents with cleft lip and palate
}

\author{
Francielle Topolski ${ }^{1}$, Rafael Boscheti de Souza ${ }^{2}$, Ademir Franco ${ }^{3}$, Osmar Aparecido Cuoghi ${ }^{1}$, \\ Luciana Reichert da Silva Assunção², Ângela Fernandes²
}

\author{
${ }^{1}$ Universidade Estadual Paulista - UNESP, Araçatuba Dental School, Department of Pediatric and Community Dentistry, Araçatuba, SP, Brazil \\ Universidade Federal do Paraná - UFPR, School of Dentistry, Department of Stomatology, Curitiba, PR, Brazil \\ ${ }^{3}$ Katholieke Universiteit Leuven, Oral Health Sciences, Area of Forensic Odontology, Leuven, Belgium
}

Received for publication: October 07, 2014 Accepted: December 16, 2014

Correspondence to: Francielle Topolski

Departamento de Odontologia Infantil e Social Disciplina de Ortodontia Preventiva - Faculdade de Odontologia de Araçatuba - UNESP

Rua José Bonifácio, 1193. Vila Mendonça CEP: 16015-050 Araçatuba, SP, Brazil Phone/Fax: +55 18 3636-3236 +55 41 9168-8717, +55 18 99600-8717 E-mail: frantopolski@gmail.com

\begin{abstract}
Aim: To evaluate the dental development of Brazilian children and adolescents with cleft lip and palate. Methods: The sample consisted of 107 panoramic radiographs of children and adolescents with cleft lip and/or palate (cleft group) and 107 panoramic radiographs of children and adolescents without cleft lip and/or palate (control group), with chronological ages ranging from 6 to 15 years, matched in gender and chronological age within 60 days. Radiographs were digitized and masked and dental age was assessed using the method described by Demirjian et al. (1973). Three trained examiners conducted the assessments. Each examiner evaluated the radiographs three times. Data were statistically analyzed using non-parametric tests and univariate linear regression $(p<0.05)$. Results: The dental age was overestimated in relation to the chronological age in both groups $(p<0.0001)$. Compared to the control group, there was a delay in the dental age in the cleft group of 0.17 years ( 2.1 months). However, no statistically significant difference in the dental age between the cleft and the control group was found even when considering the different cleft types $(p=0.152)$. Conclusions: There was no statistically significant difference in the dental age between the cleft and the control groups. The evaluation of dental development in individuals with cleft lip and palate should be approached in the same way as in individuals without clefts, with a focus on the individualization of diagnosis and treatment planning.
\end{abstract}

Keywords: cleft lip; cleft palate; dentition, permanent.

\section{Introduction}

Cleft lip and palate is the most common congenital malformation of the face. This morphologic deformity affects 1 in every 1000 births $^{1}$ and impacts on quality of life. Specifically, cleft lip and palate compromise dentomaxillofacial aesthetics and function culminating in psychosocial disorders ${ }^{1-2}$. In this context, treatment is challenging and requires a continuous multidisciplinary approach ${ }^{2-5}$.

Mostly, orthodontic treatment is essential for rehabilitation of aesthetics and function of cleft lip and palate children. However, this treatment is often prolonged due to the complexity of the situation. In order to simplify the treatment approach, accurately knowing the time for orthodontic intervention, assessment of dental maturity is of great clinical relevance ${ }^{2}$. Additionally, information concerning the ideal time for alveolar bone grafting is obtained in forehand, enabling a better surgical planning ${ }^{4,6}$.

In accordance to the clinical relevance of assessing dental maturity in cleft 
lip and palate patients, several authors reported outcomes of investigations in specific populations, such as North Americans $^{7-10}$ and Dutch ${ }^{11-13}$. Howerer, the accessment of dental maturity in cleft lip and palate patients was not performed in a Brazilian population up to the present date. Based on that, the present study aims to compare the dental development of Brazilian cleft lip and palate patients with a control group paired by chronological age and gender, contributing for further clinical diagnosis and surgical planning.

\section{Materials and methods}

\section{Sample Selection}

The study was approved by the Ethics Committee of the Division of Health Sciences, Federal University of Paraná (Curitiba, PR, Brazil). The sample consisted of 107 panoramic radiographs of children and adolescents with cleft lip and/or palate (cleft group) and 107 panoramic radiographs of children and adolescents without cleft lip and/or palate (control group), with chronological ages ranging from 6 to 15 years, matched in gender and chronological age within 60 days.

The cleft group sample was obtained from the analysis of medical records and panoramic radiographs of 160 patients with cleft lip and/or palate in a specialized treatment center. Patients presenting incomplete medical records, syndromes, systemic diseases, dental agenesis or missing permanent teeth in the mandibular left hemiarch, low-quality panoramic radiographs, and age below 6 years old or over 15 years old at the radiographic exam date were excluded from the study.

The control group consisted of the data of medical records and panoramic radiographs of children and adolescents without cleft lip and/or palate. The exclusion criteria applied for the case group were repeated for control group sampling.

No racial or ethnic data were recorded for both case and control groups. The subjects included in the sample are mainly from the state of Paraná, located in Southern Brazil. The population of this region is mainly of European descent, but also has individuals of African and indigenous origin. Therefore, the sample is characterized by large racial and ethnic heterogeneity, like most of the Brazilian population.

\section{Scanning and Blinding}

In order to avoid potential bias during the analysis, radiographs were digitized masking the identification information, including gender and date of birth. The radiographs were digitized using a scanner (Scanjet G4050) and the software Digitalização HP (Hewlett-Packard Company, Palo Alto, CA, USA). The levels of brightness and contrast indicated by the software were respected. The used standard resolution was 150 PPI (pixels per inch), on a $100 \%$ scale. Images were saved in TIFF (Tagged Image File Format), which allows generation of high quality scanned images. To identify the digitized image files, a legend of random numbers was created. Only after completing all the analyses, the examiners had access to the original identification information. In addition, the region corresponding to the maxilla was previously cut, preventing visualization of cleft areas.

\section{Evaluation of Dental Age}

Dental age was determined using the method described by Demirjian et al. ${ }^{14}$ (1973). This method is based on the evaluation of the seven left mandibular permanent teeth, excluding the third molar. The formation of each tooth is divided into eight stages, from $\mathrm{A}$ to $\mathrm{H}$, from appearance of the first points of calcification until apex closure. Each stage of tooth development corresponds to a score, which is different for males and females. The sum of the scores of the seven evaluated teeth on a panoramic radiograph corresponds to the maturity score, represented on a scale of 0 to 100 . The score of maturity, in turn, must be converted into the dental age, using two tables - one for males and other for females.

Radiographs were evaluated in a 20 -inch computer monitor in low light environment. Three trained examiners evaluated the radiographs: an oral radiologist, an orthodontist and a dental practitioner. After studying the method and training with 30 radiographs, wich were not included in the sample, each examiner evaluated the radiographs three times with a three-day interval between assessments. An average of the maturity scores obtained in the three assessments of each examiner was calculated and then a final average from the three examiners was established. The final average of maturity scores of the three examiners was converted into the dental age of each patient.

\section{Statistical Methods}

Data were analyzed using the Statistical Package for the Social Sciences (version 15.0, SPSS Inc., Chicago, IL, USA). The outcome variable considered for statistical analysis was the dental age. As there was no normal distribution of this variable (Kolgomorov-Smirnov test, $\mathrm{p}<0.001$ ), nonparametric tests for bivariate analyses were used (chi-square and Mann-Whitney U). To compare the dental age between the cleft and control groups, Wilcoxon test was used due the dependence between observations. This test was also used for the comparison between dental age and chronological age. The relationship between dental age and chronological age in both groups was also analysed by linear regression analysis. A significance level of 5\% was adopted.

In order to test accuracy and reability of the method, the intraclass correlation coefficient was used to assess intra- and inter-examiner agreement. The test was applied to the maturity scores obtained in the assessments of the first 30 radiographs. For intra-examiner analysis, the scores of maturity achieved in each of the three assessments of each examiner were compared. For inter-examiner analysis, the averages of the scores of maturity of the three examiners were compared.

\section{Results}

\section{Error of Method}

Intra-examiner analysis showed intraclass correlation 
coefficients of $0.999,0.998$ and 0.994 . The result of the interexaminer analysis was 0.995 . These results indicate very good intra- and inter-examiner agreement.

\section{Sample Characteristics}

Of the 107 pairs of children and adolescents evaluated in the study, $68(63.6 \%)$ were males and $39(36.4 \%)$ were females. The average chronological age was 10.3 years for boys and 9.8 years for girls. Among the individuals in the case group, $72(68.2 \%)$ had cleft lip and palate (CLP), 27 $(25.2 \%)$ had cleft lip with or without cleft alveolus $(\mathrm{CL} \pm \mathrm{A})$ and $2(1.9 \%)$ had cleft palate (CP). In 6 patients $(4.7 \%)$, there was an association between $\mathrm{CL} \pm \mathrm{A}$ and $\mathrm{CP}(\mathrm{CL} \pm \mathrm{A}+$ $\mathrm{CP})$. For the CLP and the CL $\pm \mathrm{A}$, unilateral clefts were more frequent $(77 \%)$ and the left side was more often affected $(63.6 \%)$.

The distribution of cleft types by gender is shown in Table 1. CLP was the most frequent type of cleft followed by $\mathrm{CL} \pm \mathrm{A}$ for both genders. The relative frequency of CLP was higher for boys $(72.1 \%)$ than for girls $(61.5 \%)$. Oppositely, the relative frequency of $\mathrm{CL} \pm \mathrm{A}$ was higher for girls $(28.2 \%)$ than for boys $(23.5 \%)$. However, considering the CLP and $\mathrm{CL} \pm \mathrm{A}$ groups, no statistically significant difference was found between cleft type and gender $(p=0.464)$. In the $C P$ and $C L \pm A+C P$ group, the small number of individuals prevented the application of statistical tests to the variables gender and cleft type.

\section{Dental Development}

Table 2 shows the descriptive statistics for the variables chronological age and dental age in cleft and control groups. The average dental age in both the cleft and the control groups was higher than the average chronological age. Dental age, therefore, was advanced in relation to chronological age in both groups, which was statistically significant for both groups $(\mathrm{p}<0.0001)$. Specifically, the advance corresponded to 0.81 years $(9.7$ months) for the cleft group and 0.98 years (11.8 months) for the control group. This result shows a delay of 0.17 years (2.1 months) in dental age in the cleft group compared to the control group; however, this difference was not statistically significant $(p=0.152)$.

The dental ages of the cleft and control groups were also compared considering the different genders, cleft types and chronological age groups $(6-9 ; 9.1$ to $12 ; 12.1$ to 15 years). Once again, no statistically significant difference $(p>0.05)$ was found as shown in Table 3. Table 4 shows the comparison of dental development between different genders and groups (cleft and control), considering the mean of differences between the dental and chronological age. As it can be seen, there was no statistically significant difference $(\mathrm{p}>0.05)$.

A strong and positive correlation between dental age and chronological age in the control and cleft groups was observed $(\mathrm{r}=0.9348, \mathrm{p}<0.001, \mathrm{r}=0.9198, \mathrm{p}<0.001$, respectively). Linear regression was used to show how much of the variation in dental age was explained by chronological age. Estimated regression showed that chronological age alone explained $87.38 \%$ of the dental age variation in the control group (Figure 1) and $84.61 \%$ in the cleft group (Figure 2).

Table 1. Distribution of cleft types by gender

\begin{tabular}{|c|c|c|c|c|c|c|}
\hline \multirow[t]{2}{*}{ Cleft Type } & \multicolumn{2}{|c|}{ Boys } & \multicolumn{2}{|c|}{ Girls } & \multicolumn{2}{|c|}{ Boys + Girls } \\
\hline & $\mathrm{n}$ & $\%$ & $\mathrm{n}$ & $\%$ & $\mathrm{n}$ & $\%$ \\
\hline$C L \pm A^{*}$ & 16 & 23.5 & 11 & 28.2 & 27 & 25.2 \\
\hline$C L P^{* *}$ & 49 & 72.1 & 24 & 61.5 & 73 & 68.2 \\
\hline$C P * * *$ & 1 & 1.5 & 1 & 2.6 & 2 & 1.9 \\
\hline$C L \pm A+C P^{* * \star \star}$ & 2 & 2.9 & 3 & 7.7 & 5 & 4.7 \\
\hline All cleft types & 68 & 100.0 & 39 & 100.0 & 107 & 100.0 \\
\hline
\end{tabular}

Table 2. Descriptive statistics for the variables chronological age and dental age (y)

\begin{tabular}{llccc}
\hline & $\begin{array}{c}\text { Chronological } \\
\text { Age }\end{array}$ & $\begin{array}{c}\text { Dental Age } \\
\text { (Cleft Group) }\end{array}$ & $\begin{array}{c}\text { Dental Age } \\
\text { (Control Group) }\end{array}$ \\
N & 107 & 107 & 107 \\
Mean & 10.12 & 10.93 & 11.10 \\
Median & 9.90 & 10.70 & 11.00 \\
Mode & & 16.00 & 16.00 \\
Standard deviation & & $(2.42)$ & $(2.66)$ & $(2.74)$ \\
Percentiles & 25 & 8.00 & 8.50 & 8.60 \\
& 50 & 9.90 & 10.70 & 11.00 \\
& 75 & 12.10 & 13.00 & 13.50 \\
Minimum & & 6.00 & 6.80 & 6.40 \\
Maximum & 14.90 & 16.00 & 16.00 \\
\hline
\end{tabular}


Table 3. Dental age in cleft vs control group by chronological age group, gender and cleft type

\begin{tabular}{|c|c|c|c|c|c|c|c|c|}
\hline & & \multirow{2}{*}{\multicolumn{3}{|c|}{ Cleft Group }} & \multirow{2}{*}{\multicolumn{3}{|c|}{ Control Group }} & \multirow[t]{3}{*}{$\begin{array}{l}\text { Dental Age } \\
\text { Cleft vs Control }\end{array}$} \\
\hline & & & & & & & & \\
\hline & & $\mathbf{M d}^{*}$ & $\mathbf{Q}_{(25)}{ }^{* *}$ & $\mathbf{Q}_{(75)}{ }^{* *}$ & $\mathrm{Md}^{*}$ & $Q_{(25)}{ }^{* *}$ & $Q_{(75)}^{* * *}$ & \\
\hline \multirow[t]{3}{*}{ Age Group } & $6-9$ & 8.3 & 7.8 & 8.6 & 8.3 & 7.7 & 8.9 & $p=0.690^{* * * *}$ \\
\hline & $9.1-12$ & 11.1 & 10.0 & 12.3 & 11.4 & 10.5 & 12.0 & $p=0.101^{* * * *}$ \\
\hline & $12.1-15$ & 14.3 & 13.2 & 15.8 & 14.7 & 13.5 & 16.0 & $p=0.899^{* \star * \star}$ \\
\hline \multirow[t]{2}{*}{ Gender } & Boys & 10.9 & 8.5 & 12.8 & 11.1 & 8.7 & 14.1 & $p=0.162^{* \star * \star}$ \\
\hline & Girls & 10.6 & 8.3 & 13.1 & 10.9 & 8.4 & 13.2 & $p=0.557^{* * \star *}$ \\
\hline \multirow[t]{3}{*}{ Cleft Type } & $C L \pm A$ & 11.2 & 8.4 & 13.2 & 10.7 & 8.6 & 14.1 & $p=0.333^{* * * *}$ \\
\hline & CLP & 10.7 & 8.5 & 12.8 & 11.2 & 8.6 & 13.4 & $p=0.401^{\star * \star *}$ \\
\hline & $\mathrm{CP}$ & 11.9 & 7.8 & 16.0 & 12.2 & 8.4 & 16.0 & $p=0.180^{* \star * *}$ \\
\hline
\end{tabular}

*Median

** $1^{\text {st }}$ Quartile

***3rd Quartile

****Wilcoxon test

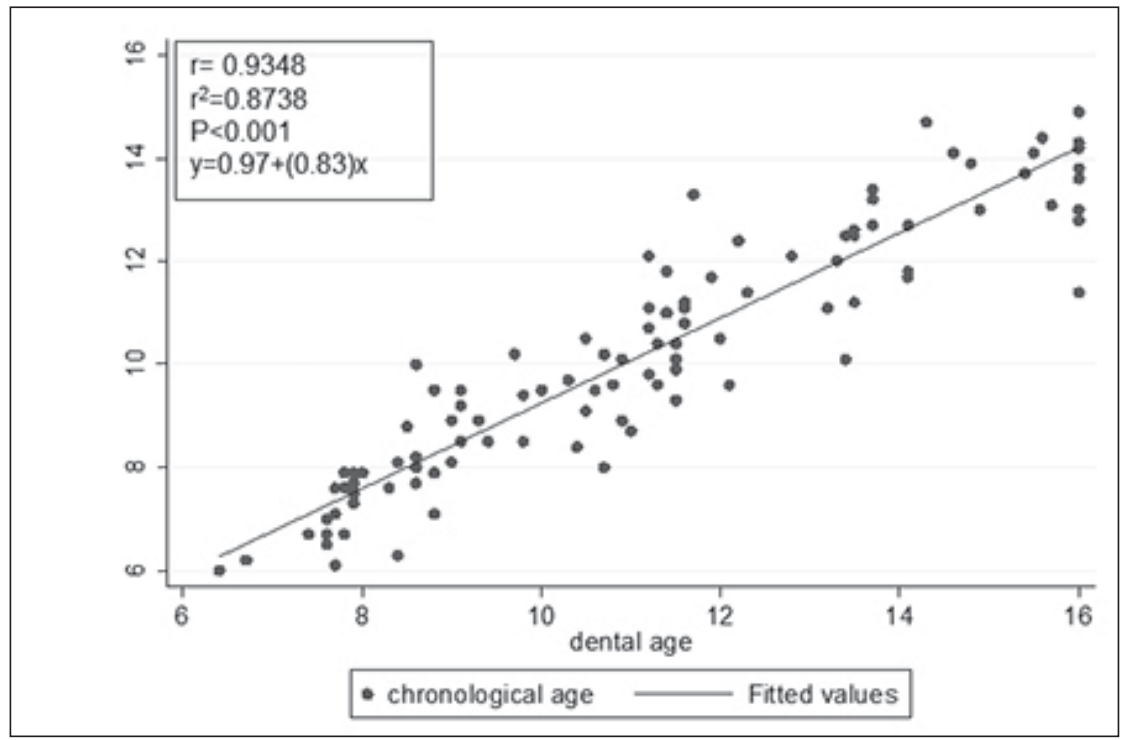

Fig. 1. Diagram showing the relationship between chronological age in years and dental age in the control group. Estimated regression equation, correlation coefficient $(r)$, coefficient of determination $\left(r^{2}\right)$ and significance level $(p)$ are also shown.

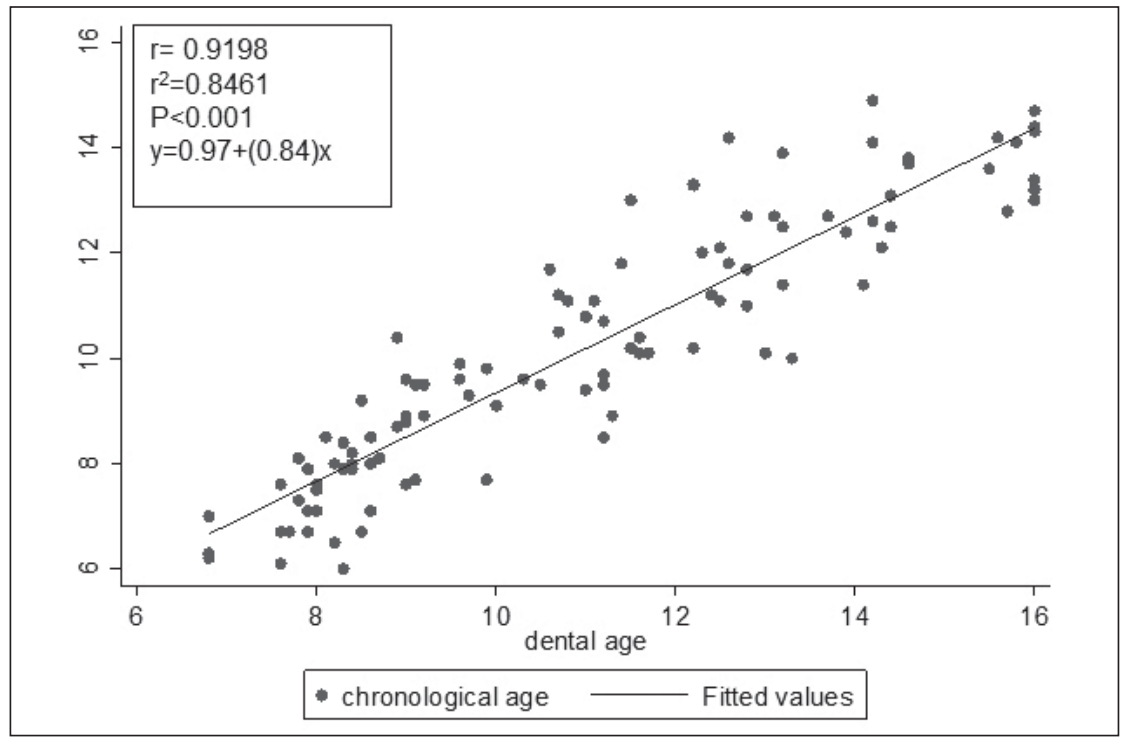

Fig. 2. Diagram showing the relationship between chronological age in years and dental age in the cleft group. Estimated regression equation, correlation coefficient $(r)$, coefficient of determination $\left(r^{2}\right)$ and significance level $(P)$ are also shown. 
Table 4. Means of the differences between dental and chronological ages

\begin{tabular}{llllll}
\hline & \multicolumn{2}{c}{ Boys } & \multicolumn{2}{c}{ Girls } & $\boldsymbol{p}^{\star *}$ \\
& Mean (y) & SD & Mean (y) & SD & \\
Cleft Group & 1.06 & $(0.80)$ & 1.02 & $(0.82)$ & 0.702 \\
Control Group & 1.16 & $(0.89)$ & 1.03 & $(0.76)$ & 0.555 \\
$\boldsymbol{p}^{*}$ & 0.415 & & 0.971 & & \\
\hline
\end{tabular}

*Wilcoxon test

**Mann-Whitney test

\section{Discussion}

In the medical literature, several authors reported population-specific results for the assessment of dental maturity in cleft lip and palate patients by comparison between cleft and control groups. In some of them, however, the cleft and the control groups were not paired by gender and/or chronological age ${ }^{7-8,11-13}$. In the present study, a control group was designed pairing patients by gender and chronological age, making the present outcomes more suitable to a population-specific survey.

Moreover, in the present investigation a larger sample is observed if compared to previous reports ${ }^{7,9-13,15-19}$ highlighting a strong reliability. In addition, contrarily to previous studies ${ }^{7-}$ 9,11-13,15,17-20 a blinding methodology was applied to avoid potential bias during the image analysis. The blinding methodology plays an important part in the study reliability, since the method of Demirjian et al. ${ }^{14}(1973)$ presents a certain degree of subjectivity. Some teeth are in intermediate stages of development (e.g. between stages D and E) potentially hampering the process of age estimation. If the examiner knows that the radiography belongs to an individual with cleft, he may tend to choose a premature stage, since the literature reports that the dental development in these individuals is delayed. Therefore the importance of blinding is justified. Furthermore, in relation to the image analysis, it is relevant to note that three calibrated examiners performed the assessment of dental maturity. Only two previous studies ${ }^{12,17}$ report these standards to achieve greater reliability.

The method of Demirjian et al. ${ }^{14}(1973)$ was applied in the present sample, revealing a slight developmental delay of the dental age in the cleft group, therefore not statistically significant. Similarly, Eerens et al. ${ }^{16}$ (2001) reported a slight delay in the dental development of Belgian children. Despite the concordance, both studies contradict most of the previous

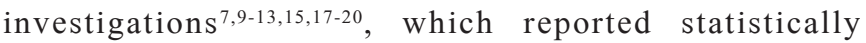
significant delays in the dental development of individuals with cleft lip and palate. Moreover, some studies lack information concerning the performed methodology $\mathrm{y}^{7,15}$, hampering adequate interpretations. Yet other authors performed different methods for dental age assessment, not allowing for accurate comparisons ${ }^{11,17}$. In the study of Bindayel et al. ${ }^{19}$ (2014) no control group was used, which may compromise the reliability of the results.

Thus, the differences observed in the current study when compared with other studies that evaluated the dental development of individuals with cleft lip and palate may be explained by the applied methodological design, considering aspects such as sample size, pairing of the sample, blinding, number of examiners and number of radiograph evaluations per examiner. Moreover, ethnic and racial differences among the surveyed populations may have contributed to these differences.

Furthermore, in the present study individuals with agenesis in mandibular left hemiarch were excluded. The etiologic factors of delayed dental development of individuals with clefts seem to be the same factors responsible for the occurrence of dental anomalies in these individuals, as well as for the manifestation of the cleft itself ${ }^{7,16,21}$. Moreover, the delay tends to be more pronounced in individuals with agenesis ${ }^{21}$. This may explain the absence of delayed dental development in the individuals with cleft lip and palate observed in this study. We excluded individuals who had agenesis and a possible greater probability of changes in dental development. On the other hand, Ruiz-Mealin et al. ${ }^{22}$ (2012) observed delays in the dental development of subjects with agenesis and without any syndrome or medical condition (meaning without clefts). This may suggest that delayed dental development is more associated with the presence of agenesis than with cleft lip and palate.

There was a strong correlation between dental age and chronological age in the control and cleft groups. However, the dental age was advanced in relation to chronological age in the cleft and control groups with a statistically significant difference for both groups. This finding was also reported by other studies ${ }^{23-25}$ that aimed to estimate the chronological age by assessing the dental age using the method described by Demirjian et al. ${ }^{14}$ (1973). These results demonstrate that this method tends to overestimate the dental age when applied to different populations.

For the present study, however, this fact was not relevant, since the goal was not to estimate the chronological age from the dental age. The Demirjian et al..$^{14}$ (1973) method was used with the purpose of comparing the dental age of individuals from cleft and control groups with the same chronological age.

No statistically significant difference in dental age between the cleft and control groups was found even when considering the different genders, cleft types and chronological age groups separately. Therefore, one can conclude that the analysis of dental development in individuals with cleft lip and palate should have the same approach used for individuals without clefts, with a focus on the individualization of diagnosis and treatment planning.

\section{Acknowledgement}

This work was supported by a Brazilian research funding agency (CAPES).

\section{References}

1. World Health Organization. Global strategies to reduce the health-care burden of craniofacial anomalies. Geneva: The Organization; 2002. 
2. Freitas JAS, Garib DG, Oliveira TM, Lauris RCMC, Almeida ALPF, Neves LT, et al. Rehabilitative treatment of cleft lip and palate: experience of the Hospital for Rehabilitation of Craniofacial Anomalies - USP (HRACl USP) - Part 2: pediatric dentistry and orthodontics. J Appl Oral Sci. 2012; 20: 268-81.

3. David DJ, Smith I, Nugent M, Richards C, Anderson PJ. From birth to maturity: a group of patients who have completed their protocol management. Part III. Bilateral cleft lip-cleft palate. Plast Reconstr Surg. 2011; 128: 475-84.

4. Toscano D, Baciliero U, Gracco A, Siciliani G. Long-term stability of alveolar bone grafts in cleft palate patients. Am J Orthod Dentofacial Orthop. 2012; 142: 289-99.

5. Farronato G, Kairyte L, Giannini L, Galbiati G, Maspero C. How various surgical protocols of the unilateral cleft lip and palate influence the facial growth and possible orthodontic problems? Which is the best timing of lip, palate and alveolus repair? Literature review. Stomatologija. 2014; 16: 53-60.

6. Osawa T, Omura S, Fukuyama E, Matsui Y, Torikai K, Fujita K. Factors influencing secondary alveolar bone grafting in cleft lip and palate patients: prospective analysis using CT image analyzer. Cleft Palate Craniofac J. 2007; 44: 286-91.

7. Bailit HL, Doykos JD, Swanson LT. Dental development in children with cleft palates. J Dent Res. 1968; 47:664.

8. Loevy HT, Aduss $\mathrm{H}$. Tooth maturation in cleft lip, cleft palate, or both. Cleft Palate J. 1988; 25: 343-7.

9. Pham AND, Seow WK, Shusterman S. Developmental dental changes in isolated cleft lip and palate. Pediatr Dent. 1997; 19: 109-13.

10. Borodkin AF, Feigal RJ, Beiraghi S, Moller KT, Hodges JS. Permanent tooth development in children with cleft lip and palate. Pediatr Dent. 2008; 30: 408-13.

11. Brouwers HJM, Kuijpers-Jagtman AM. Development of permanent tooth length in patients with unilateral cleft lip and palate. Am J Orthod Dentofacial Orthop. 1991; 99: 543-9.

12. Heidbüchel KLWM, Kuijpers-Jagtman AM, Ophof R, Van Hooft RJM. Dental maturity in children with a complete bilateral cleft lip and palate. Cleft Palate Craniofac J. 2002; 39: 509-12.

13. Huyskens RWF, Katsaros C, Van't Hof MA, Kuijpers-Jagtman AM. Dental age in children with a complete unilateral cleft lip and palate. Cleft Palate Craniofac J. 2006; 43: 612-5.

14. Demirjian A, Goldstein H, Tanner JM. A new system of dental age assessment. Hum Biol. 1973; 45: 211-27.

15. Harris EF, Hullings JG. Delayed dental development in children with isolated cleft lip and palate. Arch Oral Biol. 1990; 35: 469-73.

16. Eerens K, Vlietinck R, Heidbüchel K, Olmen AV, Derom C, Willems G, et al. Hypodontia and tooth formation in groups of children with cleft, siblings without cleft, and nonrelated controls. Cleft Palate Craniofac J. 2001; 38: 374-8.

17. Mitsea AG, Spyropoulos MN. Premolar development in Greek children with cleft lip and palate. Quintessence Int. 2001; 32: 639-46.

18. Tan ELY, Yow M, Kuek MC, Wong HC. Dental maturation of unilateral cleft lip and palate. Ann Maxillofac Surg. 2012; 2: 158-62.

19. Bindayel NA, AlSultan MA, ElHayek SO. Timing of dental development in Saudi cleft lip and palate patients. Saudi Med J. 2014; 35: 304-8.

20. Lai MC, King NM, Wong HM. Dental development of Chinese children with cleft lip and palate. Cleft Palate Craniofac J. 2008; 45: 289-96.

21. Ranta R. A review of tooth formation in cleft lip/palate. Am J Orthod Dentofacial Orthop. 1986; 90: 11-8.

22. Ruiz-Mealin EV, Parekh S, Jones SP, Moles DR, Gill DS. Radiographic study of delayed tooth development in patients with dental agenesis. Am J Orthod Dentofacial Orthop. 2012; 141: 307-14.

23. Koshy S, Tandon S. Dental age assessment: the applicability of Demirjian's method in south Indian children. Forensic Sci Int. 1998; 94: 73-85.

24. Willems G, Olmen AV, Spiessens B, Carels C. Dental age estimation in Belgian children: Demirjian's technique revisited. J Forensic Sci. 2001; 46: 893-5.
25. Kýrzýoðlu Z, Ceyhan D. Accuracy of different dental age estimation methods on Turkish children. Forensic Sci Int. 2012; 216: 61-7. 